\title{
A CIÊNCIA DA INFORMAÇÃO NO BRASIL E SUA FRENTE DE PESQUISA: estudo cienciométrico sob a ótica da institucionalização da pesquisa científica (1995-2005) ${ }^{1}$ \\ THE INFORMATION SCIENCE IN BRAZIL AND ITS RESEARCH FRONT: scienciometric study under the optics of the institutionalization of the scientific research (1995-2005)
}

\author{
Murilo Artur Araújo da Silveira \\ Mestre em Ciência da Informação - PUC-Campinas \\ Rogério Eduardo Rodrigues Bazi \\ Doutor em Ciências da Comunicação (USP)
}

Docente do Programa de Pós-graduação em Ciência da Informação - PUC-Campinas

\begin{abstract}
Resumo
Identificação dos os principais personagens científicos ligados à Ciência da Informação brasileira, através do periódico Ciência da Informação. Tem como objetivo investigar a institucionalização social da Ciência da Informação com base nas citações realizadas nos artigos científicos do periódico Ciência da Informação, no período de 1995 a 2005. Utiliza o método cienciométrico, através da análise de citações e representações cartográficas. Os resultados são apresentados segundo os grupos de trabalho da ANCIB. Tece considerações sobre os resultados e sugere modificações nas indicações das citações.
\end{abstract}

Palavras-chave: Ciência da Informação. Frente de Pesquisa. Cienciometria. Periódico Ciência da Informação. Estudos de Citação. Institucionalização da Pesquisa Científica.

\section{INTRODUÇÃO}

Uma disciplina científica é um todo organizado que congrega elementos específicos. A sua constituição se concretiza a partir das formas de operação com os seus objetos, teorias, metodologias, problemas, instrumentos e estruturas formais e informais.

O nível das operações realizadas por uma disciplina determina o grau de institucionalização da pesquisa científica. Whitley (1980) aponta que uma disciplina científica se consolida na particularidade de suas estruturas e realidades. Para Parlemiti e Polity (2002, p. 95) a institucionalização "se coloca em termos de processos e se inscreve no eixo diacrônico". Assim, pode-se entender que a institucionalização de uma disciplina se dá a partir de diálogos e atividades empreendidas pelos atores e instituições em torno das estruturas teóricometodológicas consensuais que se vinculam à disciplina científica.

\footnotetext{
${ }^{1}$ Trabalho baseado na dissertação de mestrado: Rede de textos científicos: um estudo sob a ótica da institucionalização da Ciência da Informação no Brasil, defendida no Programa de Pós-graduação em Ciência da Informação da PUC-Campinas.

Enc. Bibli: R. Eletr. Bibliotecon. Ci. Inf., Florianópolis, n. 26, $2^{\circ}$ sem.2008
} 
Whitley (1974) propõe que a institucionalização da pesquisa científica de uma disciplina científica pode ser visualizada a partir de duas vertentes: a cognitiva e a social. Em linhas gerais, a institucionalização cognitiva relaciona-se aos aspectos epistemológicos, teóricos e metodológicos de uma disciplina científica. Por sua vez, a institucionalização social pode ser observada pelas formas como a disciplina legitima e desenvolve as instâncias organizacionais que se relacionam a ela.

Um elemento importante na materialização da institucionalização cognitiva e social de um campo é o artigo científico. Mueller (1999) afirma que os periódicos e, por conseguinte, os artigos científicos podem ser avaliados tendo como premissa a sua representatividade enquanto elemento disseminador de conhecimentos científicos. Também aponta que os periódicos, os autores, o artigo, as citações ou referências bibliográficas aos seus artigos ou por ele referidas são unidades de análise relevantes quando se quer avaliar uma ciência e suas contribuições (MUELLER, 1999).

Estudar os pesquisadores e suas contribuições é salutar para uma disciplina científica, pois se pode conhecer seus principais atores, assim como o impacto de suas publicações no universo que estão inseridos. Nesse horizonte, a oportunidade que surge é investigar as citações que são efetuadas aos pesquisadores e às suas publicações. Price (1965) ao analisar o comportamento, a distribuição e a incidência de citações, descreve que ao longo dos anos um número muito pequeno de citações continua a ser freqüente, originando um núcleo de concentração de pesquisadores e textos. O núcleo de pesquisadores e textos citados se denomina frente de pesquisa de uma disciplina científica, ou seja, o conjunto de pesquisadores e textos que pelo seu valor e sua contribuição continuam a serem citados.

Dessa forma, o presente artigo busca destacar a frente de pesquisa em Ciência da Informação no Brasil, com base nas citações realizadas nos artigos científicos do periódico Ciência da Informação, no período de 1995 a 2005. Trata-se de um estudo de citação e se insere nos estudos de institucionalização social da pesquisa científica, objetivando identificar os personagens científicos e lhes atribuir os devidos créditos, no mesmo instante que possibilita à Ciência da Informação uma melhor compreensão dos seus caminhos percorridos.

Este trabalho se justifica em razão dos inúmeros registros na literatura brasileira que chamam a atenção para a inexistência de trabalhos que evidenciem o estágio atual da Ciência da Informação (GOMES, 2006; PINHEIRO, 2005; OLIVEIRA; SANTANA, 2005; QUEIROZ; NORONHA, 2004). Outra justificativa decorre do fato de se obter um maior entendimento das operações cognitivas e sociais que se processam quando se determina uma frente de pesquisa, uma vez que esta determinação está condicionada a domínios específicos de uma disciplina científica. Portanto, o estudo em questão busca sinalizar a frente de pesquisa que se formou no período de 1995 a 2005, nos domínios de conhecimento da Ciência da Informação brasileira, ilustrando os caminhos percorridos por ela no seu processo de institucionalização.

\section{MÉTODO E PROCEDIMENTOS METODOLÓGICOS}

O corpus desta contribuição é composto pelos artigos científicos publicados no periódico Ciência da Informação de 1995 a 2005, disponíveis em meio eletrônico e as referências contidas no final dos artigos contemplados. O número de artigos científicos é de 386 e o de referências, 7.516.

Enc. Bibli: R. Eletr. Bibliotecon. Ci. Inf., Florianópolis, n. 26, $2^{\circ}$ sem.2008 
O periódico Ciência da Informação foi escolhido com base em estudos anteriores que apontam a representatividade dele para o campo. Alia-se a esta justificativa o fato deste periódico possuir mais de 25 anos de atividades ininterruptos, colocando-se assim como um dos periódicos de maior visibilidade e impacto da Ciência da Informação. Conta com a participação de expressivos e experientes pesquisadores em seu comitê editorial e suas políticas e normas editoriais são claras e objetivas. Seu propósito maior é veicular trabalhos originais relacionados aos processos de produção, organização, disseminação, recuperação e uso da informação, na perspectiva das teorias e práticas da Ciência da Informação.

Martyn (1979, p. 69 citado por TARGINO; GARCIA, 2000, p, 106) afirma que "a essência da ciência está num número muito pequeno de periódicos, e a maioria dos periódicos representa, de fato, a minoria da literatura científica”. Os trabalhos de Cunha (1985), Foresti (1990) e Silveira e Moura (2006) mostram um panorama da época sobre a área, e confirmam a posição de destaque que o periódico Ciência da Informação possui perante a comunidade científica brasileira.

Adotou-se o método cienciométrico, a partir da análise de citações, da análise de assunto e de representações cartográficas. O método cienciométrico para Macias-Chapula (1998, p. 134) “é o estudo dos aspectos quantitativos da ciência enquanto uma disciplina ou atividade econômica. A cientometria é um segmento da sociologia da ciência, sendo aplicada no desenvolvimento de políticas científicas [...]”. Bufrem e Prates (2005) conceituam cienciometria como o método quantitativo utilizado no estudo de atividades científicas e/ou técnicas, do ponto de vista de sua produção ou comunicação. "Tenta medir os incrementos de produção e produtividade de uma disciplina, de um grupo de pesquisadores de uma área, a fim de delimitar o crescimento de determinado ramo do conhecimento" (TAGUE-SUTICKFFE citado por VANTI, 2002, p. 154).

Os procedimentos metodológicos utilizados por esta pesquisa são descritos a seguir:

- Identificação dos volumes e dos números do período de 1995 a 2005 do periódico Ciência da Informação;

- $\quad$ Captura dos arquivos em formato .pdf disponibilizados na página do periódico;

- Definição e formatação da base de dados, assim como dos campos e de suas estruturas no software Microsoft Word;

- $\quad$ Alimentação da base a partir dos dados extraídos dos arquivos em formato .pdf;

- Eliminação das inconsistências dos dados e dos erros de digitação, através da função Substituir do software Microsoft Word;

- Definição e criação dos gráficos a partir do software Microsoft Excel;

- Discussão dos resultados, tendo por base as representações cartográficas que foram elaboradas.

\section{RESULTADOS E DISCUSSÃO}

A média de citações por artigo, do conjunto analisado foi de 19,47. Esse valor é superior ao preconizado por Price (1965), que aponta que um artigo, em média, tem 15 citações. Todavia, cabe o registro de que 14 artigos apresentaram suas citações em forma de notas bibliográficas e que 10 não apresentaram a lista de referências. Caso essas situações não tivessem acontecido, é bem provável que a média de citações por artigo do periódico analisado seria 
maior. No entanto, salienta-se que Price (1965) menciona que a distribuição das citações é irregular, esclarecendo que as incidências de citações por artigos sofrem variações de acordo com o tipo de contribuição científica veiculada nos periódicos.

O número de autores citados no período compreendido pela pesquisa foi de 5.054. Deste total, 3.752 autores foram citados uma vez, 675 autores duas vezes e 254 autores três vezes, totalizando 4.681 autores. Estes 4.681 autores correspondem 92,61\% dos autores citados, evidenciando que uma parte muito pequena das citações se concentra em poucos pesquisadores. Tal situação é descrita na pesquisa de Price (1965), em que o autor detectou a existência de um grupo de autores que, por suas idéias e contribuições ao corpus de uma disciplina, se destacam recebendo assim, mais citações. O Gráfico 1 ilustra o grupo de autores mais citados nos artigos do periódico Ciência da Informação, no período de 1995 a 2005.

\section{GRÁFICO 1: AUTORES MAIS CITADOS}

O Gráfico 1 representa o ranking de ocorrência e freqüência dos autores mais citados nos artigos veiculados pelo periódico Ciência da Informação. $\mathrm{O}$ valor de ocorrência corresponde ao número de citações recebidas por cada autor. $\mathrm{O}$ valor de freqüência representa o número 
isolado de citações efetuadas por artigo para um determinado autor. Assim, um artigo que citou um mesmo autor três vezes, o valor da ocorrência de citação deste autor será três e o de freqüência será um.

Antes de iniciar as análises, é importante registrar que o item Sem Autoria representa as citações aos autores desconhecidos, não identificados e organizadores de livros. A maioria das citações de autores desconhecidos advém das páginas de internet; já os itens não identificados se referem às referências que não registram os elementos corretamente; e por fim os organizadores de livros que para fins de estudos de citação não são autores.

O autor mais citado no período analisado foi Pierre Levy, com 44 citações por 28 artigos, vindo em seguida, F. W. Lancaster e Tefko Saracevic, com 41 e 38 citações por 30 e 24 artigos, respectivamente, para então aparecer o primeiro brasileiro da lista Aldo de Albuquerque Barreto, citado 33 vezes por 26 artigos.

Do elenco de autores citados, verifica-se a presença de autores que não são considerados como personagens científicos da Ciência da Informação, mas que pelo valor de suas contribuições científicas foram citados, independentemente de qual ciência se dedicam. Notase a presença de muitos cientistas estrangeiros e de apenas seis brasileiros ligados a Ciência da Informação. O panorama apontado pela análise salienta a desigualdade quantitativa e qualitativa no número de pesquisas que são desenvolvidas em outros países e no Brasil, evidenciando que o volume das pesquisas significativas para a Ciência da Informação são desenvolvidas em outros países. Contudo, cabe ressaltar que a diferença entre a incidência de autores brasileiros e de outros não é tão expressiva, revelando que as pesquisas e atividades de informação no país crescem em quantidade e qualidade.

Esta contribuição também concentrou suas análises nas relações entre as temáticas e os autores citados. Optou-se por apresentar os autores mais citados por temáticas, tendo como referência os Grupos de Trabalho da ANCIB de forma individualizada. Os grupos que servem de referência para a apresentação das representações cartográficas são arrolados pelo Quadro 1 .

\begin{tabular}{|c|c|}
\hline Grupo & Descrição do Grupo \\
\hline GT 1 & Estudos Históricos e Epistemológicos da Informação \\
\hline GT 2 & Organização e Representação do Conhecimento \\
\hline GT 3 & Mediação, Circulação e Uso da Informação \\
\hline GT 4 & Gestão da Informação e do Conhecimento nas Organizações \\
\hline GT 5 & Política e Economia da Informação \\
\hline GT 6 & Informação, Educação e Trabalho \\
\hline GT 7 & Produção e Comunicação da Informação em CT\&I \\
\hline
\end{tabular}

Como expresso por Price (1965), a frente de pesquisa abarca um número reduzido de pesquisadores citados, formando assim um núcleo. Então, o número de ocorrência de citações aos pesquisadores foi de quatro, haja visto que do conjunto total, 92,61 de citações a pesquisadores contempla uma a três citações. 
Assim, a primeira representação cartográfica refere-se aos autores mais citados em temáticas ligadas ao GT1 (Estudos históricos e epistemológicos da informação), a partir do Gráfico 2.

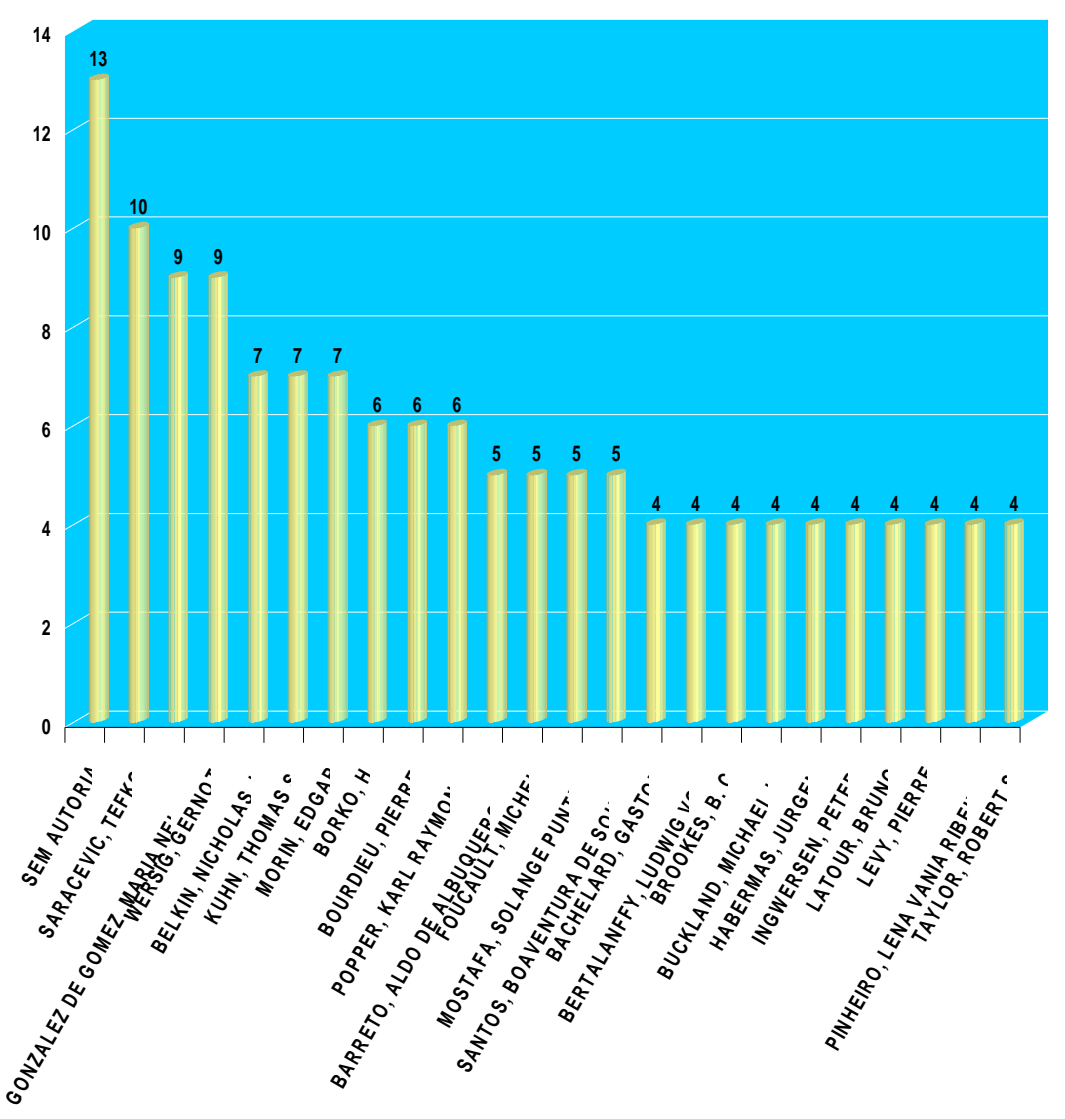

\section{GRÁFICO 2: AUTORES MAIS CITADOS DO GT1}

O item Sem Autoria aparece no primeiro lugar deste grupo, seguido por Tefko Saracevic com dez citações e depois Maria Nélida Gonzalez de Gómez com nove citações. Desse grupo, destacam-se personagens científicos que não possuem um vínculo com a Ciência da Informaçao como Thomas S. Kuhn, Edgar Morin, Pierre Bourdieu e Jurgen Habermas.

Entre os autores estrangeiros ligados à Ciência da Informação têm-se: Gernot Wersig, Nicholas J. Belkin, Michael K. Buckland e Peter Ingwersen. A presença dos autores no grupo pode ser explicada pelo fato que algumas de suas contribuições são consideradas clássicas e de grande repercussão nos cursos de graduação e pós-graduação brasileiros, versando sobre os aspectos teóricos e epistemológicos da área.

Entre os autores brasileiros citados nos artigos com assuntos voltados para os conhecimentos teórico-epistemológicos da Ciência da Informação, destacam-se Aldo de Albuquerque Barreto, Solange Puntel Mostafa e Lena Vânia Ribeiro Pinheiro.

O Gráfico 3 exibe os autores mais citados em temáticas ligadas à organização, tratamento e recuperação da informação, representadas pelo GT2 (Organização e representação do conhecimento). 


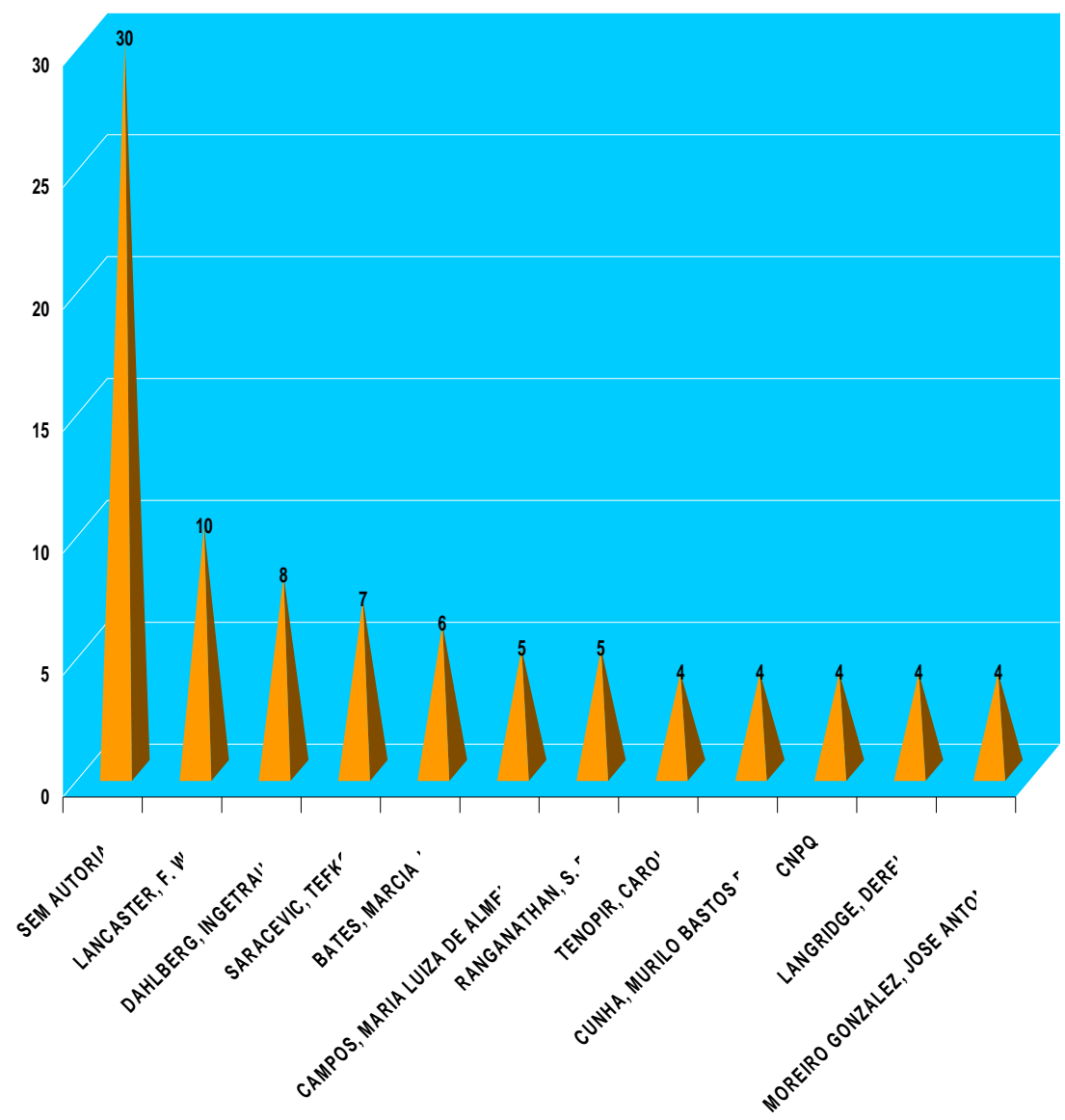

\section{GRÁFICO 3: AUTORES MAIS CITADO DO GT2}

O Gráfico 3 coloca em evidência a primeira posição para o item Sem Autoria. Assim como no grupo anterior, este item assume a primeira colocação no grupo de trabalho. O autor $F$. $W$. Lancaster surge em segundo lugar com 10 citações, seguido por Ingetraut Dahlberg com 8 citações. Em seguida, Tefko Saracevic com 7 citações. Nota-se que é a segunda ocorrência do autor em dois grupos de trabalho distintos.

A representação cartográfica acima indica que há forte incidência de autores estrangeiros considerados influentes nos processos de organização e tratamento da informação, como $S$. $R$. Ranganathan e Derek Langridge. Alia-se a este grupo de atores científicos estrangeiros, Carol Tenopir e José Antonio Moreiro Gonzalez.

Considerando até a freqüência de 4 citações, os autores Maria Luiza de Almeida Campos e Murilo Bastos da Cunha são os únicos brasileiros que figuram entre os mais mencionados. Destaque ainda para o CNPq enquanto autor citado nos trabalhos deste grupo.

A seguir, destacam-se os autores mais citados nos limites de cobertura temática do GT3 (Mediação, circulação e uso da informação) que focaliza assuntos ligados à disseminação e uso da informação, conforme mostra o Gráfico 4. 


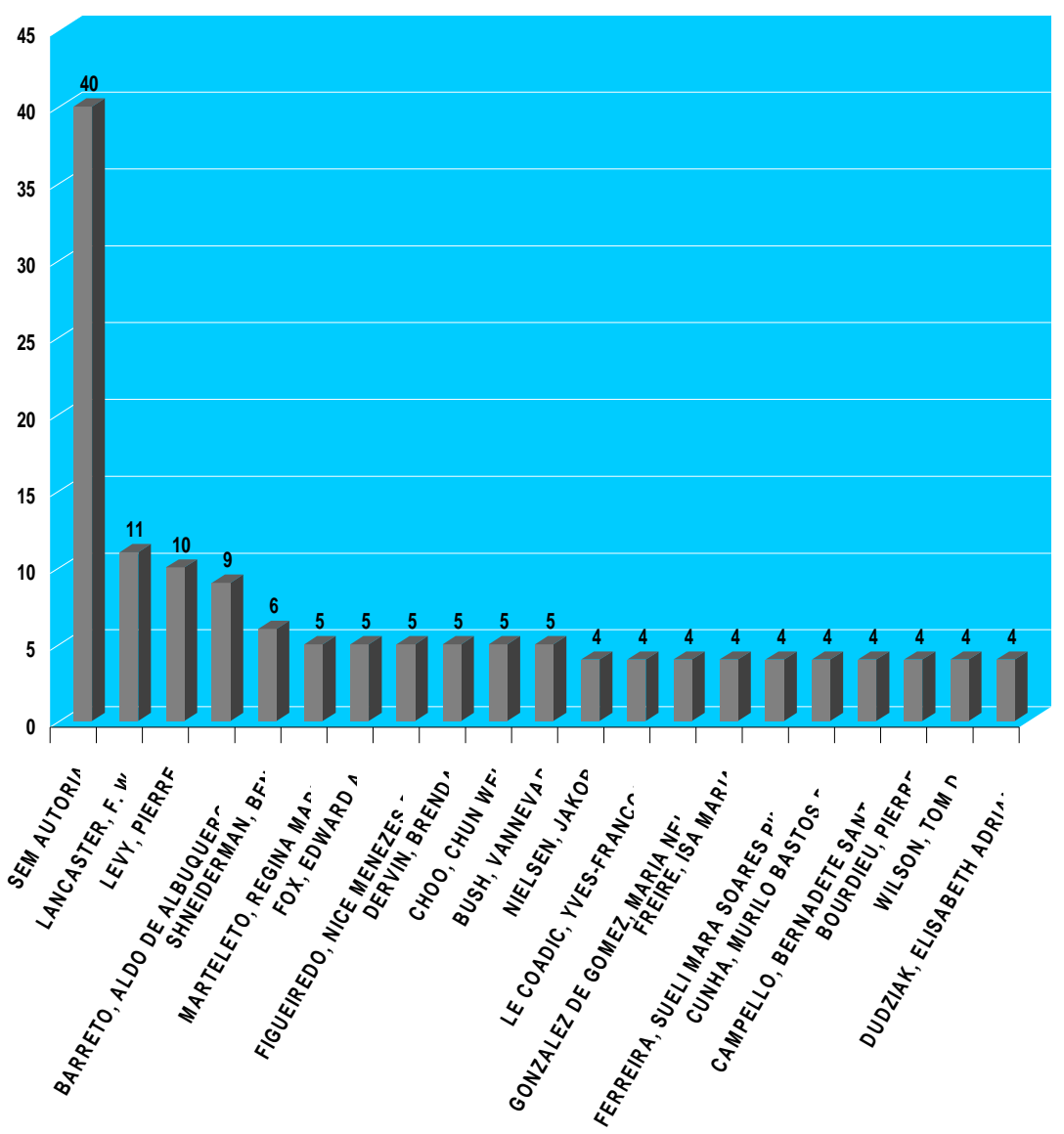

\section{GRÁFICO 4: AUTORES MAIS CITADOS NO GT3}

A representação cartográfica acima apresenta o item Sem Autoria na primeira posição com 40 citações neste grupo, sendo sua terceira ocorrência na análise realizada até agora. Logo em seguida, surge F. W. Lancaster com 11 citações e Pierre Levy com 10 citações. É a segunda ocorrência dos dois autores nos três grupos de trabalhos analisados. Na quarta colocação está o primeiro autor nacional com 6 citações, Aldo de Albuquerque Barreto, sendo também sua segunda ocorrência entre os grupos apresentados até o momento.

Desta representação percebe-se uma maior incidência de autores brasileiros em relação aos autores estrangeiros. Do total, 11 são os autores estrangeiros e oito são autores nacionais. Dos autores brasileiros mais citados, destaca-se Regina Maria Marteleto, Nice Menezes de Figueiredo, Isa Maria Freire, Sueli Mara Soares Pinto Ferreira, Bernadete Santos Campello e Elisabeth Adriana Dudziak. Evidencia-se também Maria Nélida Gonzalez de Gómez e Murilo Bastos da Cunha pela segunda ocorrência até então.

Dentre os personagens estrangeiros mais mencionados neste grupo de trabalho, tem-se Ben Shneiderman, Edward A. Fox, Brenda Dervin, Chun Wei Choo, Vannevar Bush, Nielsen Jakob, Pierre Bourdieu e Tom D. Wilson.

O Gráfico 5, expresso a seguir, apresenta os autores mais citados no GT4 (Gestão da informação e do conhecimento nas organizações), que direciona sua atuação aos estudos de gestão da informação e do conhecimento. 


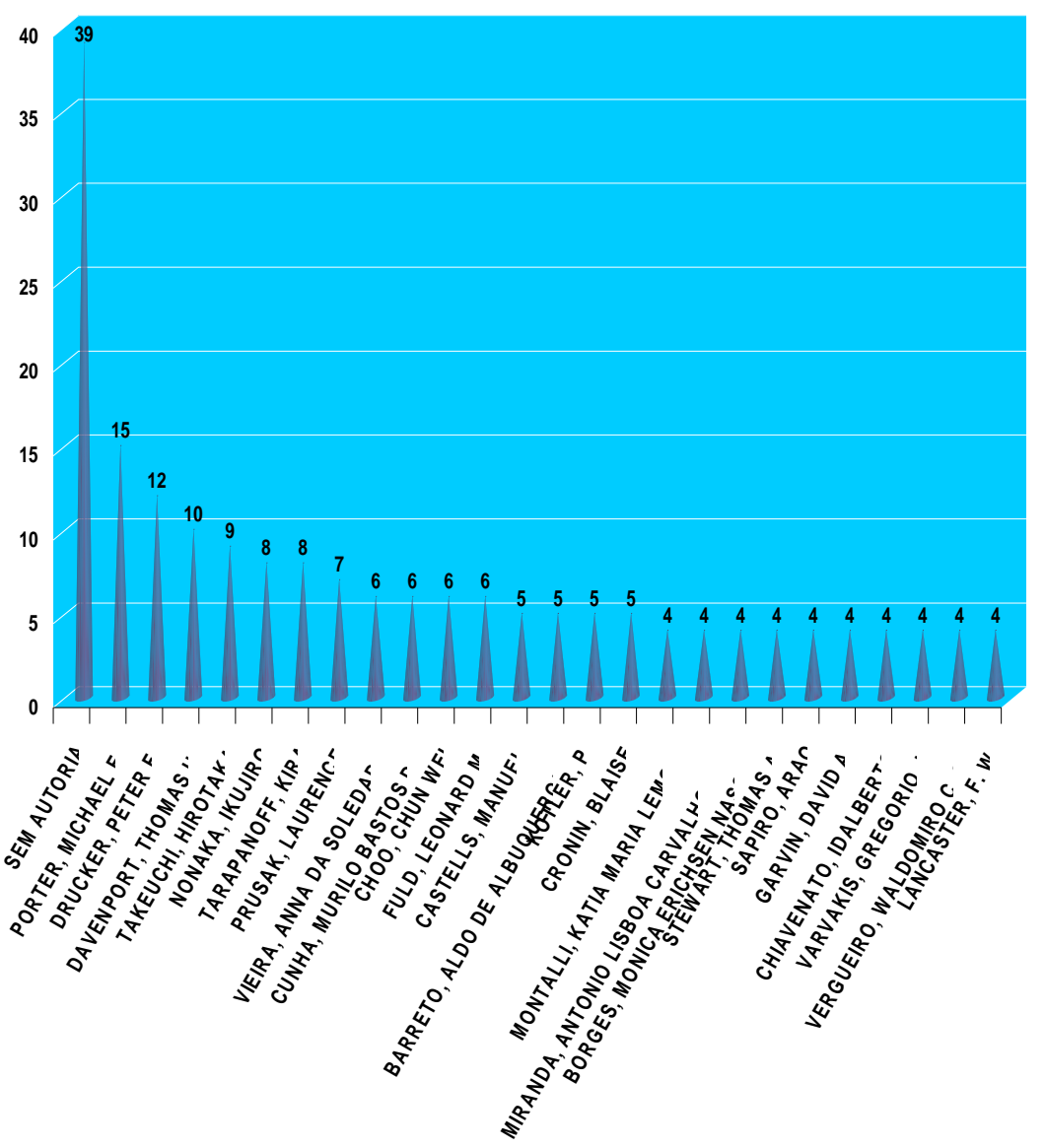

GRÁFICO 5: AUTORES MAIS CITADOS NO GT4

O Gráfico 5 apresenta o ranking dos autores mais citados nos artigos que foram categorizados no grupo em questão, mostrando o item Sem Autoria na primeira colocação, sendo sua quarta ocorrência na primeira posição e nos quatros grupos de trabalhos avaliados. Os autores Michael E. Porter e Peter F. Drucker assumem a segunda e terceira posições com 15 e 12 citações, nessa ordem. O autor brasileiro mais bem posicionado no rol dos mais citados é Kira Tarapanoff, com oito citações.

Neste grupo, percebe-se a presença de muitos autores estrangeiros ligados à Ciência da Informação e outras áreas do conhecimento. Coloca-se em evidência Thomas H. Davenport, Hirotaka Takeuchi, Ikujiro Nonaka, Laurence Prusak, Chun Wei Choo e Manuel Castells. Destaque ainda pra F. W. Lancaster que já figurou em dois grupos anteriores, os GT3 e GT2, apresentando-se portanto, pela terceira vez.

Dos brasileiros citados, registra-se a presença de Anna da Soledade Vieira, Kátia Maria Lemos Montalli, Antonio Lisboa Carvalho de Miranda, Mônica Erichsen Nassif Borges, Gregório J. Varvakis e Waldomiro C. S. Vergueiro. Aldo de Albuquerque Barreto e Murilo Bastos da Cunha aparecem no grupo e contabilizam três ocorrências em quatro grupos de trabalhos analisados. 
Os autores que mais receberam menções no GT5 (Política e economia da informação) da ANCIB que se dedica aos estudos dos aspectos políticos, econômicos, sociais e culturais ligados à informação são apresentados pelo Gráfico 6.

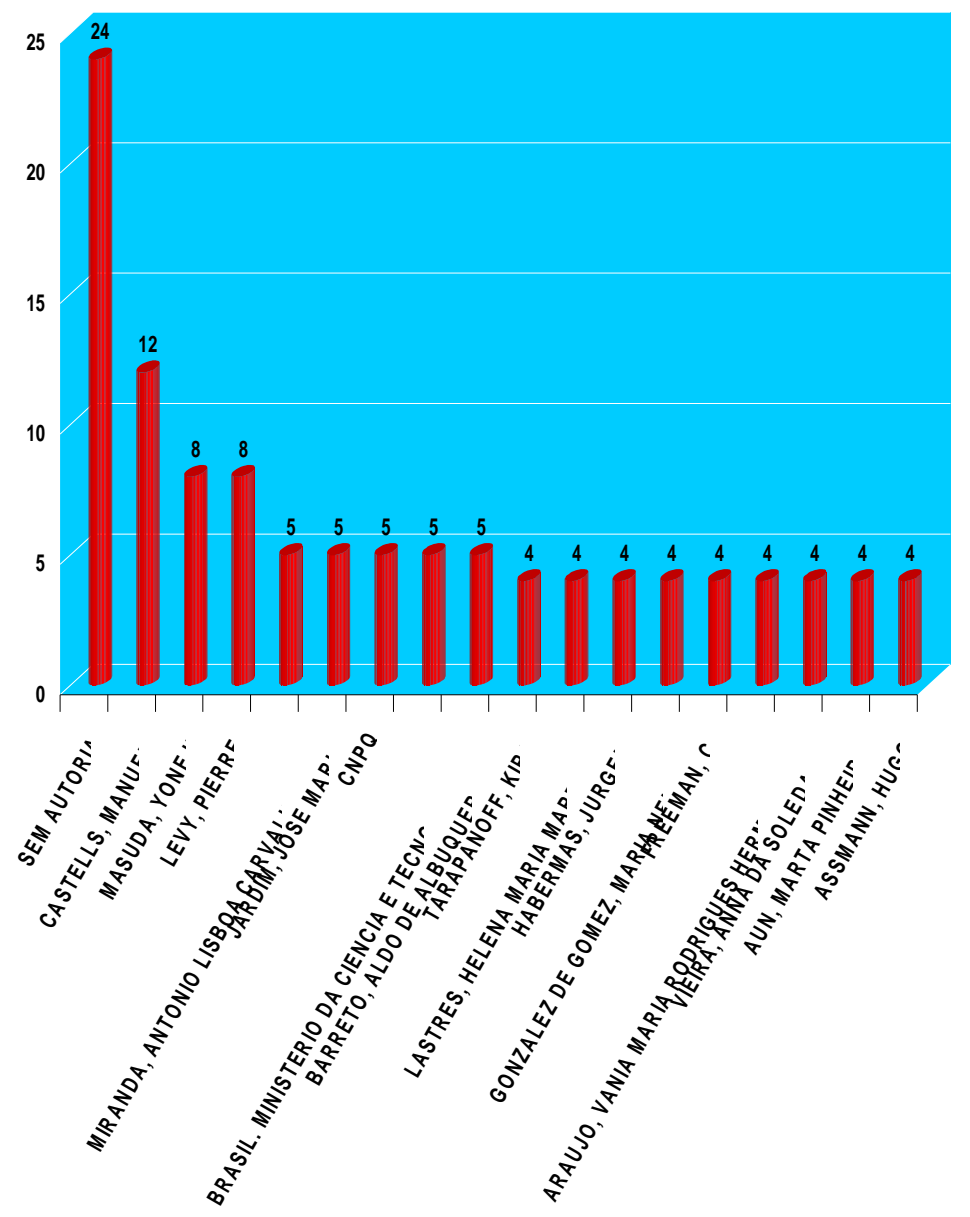

\section{GRÁFICO 6: AUTORES MAIS CITADOS NO GT5}

A primeira análise do Gráfico 6 é que o grupo de trabalho em questão é o primeiro que apresentou um número maior de pesquisadores brasileiros citados em relação aos estrangeiros. O item Sem Autoria aparece, mais um a vez, no primeiro lugar e indica Manuel Castells na segunda posição com 12 citações, e a segunda ocorrência nos cinco grupos analisados até agora. Ioneji Masuda e Pierre Levy que ocupa a terceira e quarta posição, respectivamente, com oito citações cada um, também já tiveram ocorrências em outros grupos verificados.

O primeiro brasileiro da lista dos mais mencionados é Antonio Lisboa Carvalho de Miranda com cinco citações, e registra sua segunda ocorrência até então. Outros personagens nacionais de destaque são José Maria Jardim, Helena Maria Martins Lastres, Vânia Maria Rodrigues Hermes de Araújo e Marta Pinheiro Aun. Já Aldo de Albuquerque Barreto, Kira Tarapanoff, Maria Nélida Gonzalez de Gómez e Anna da Soledade Vieira aparecem entre os mais citados e mais de uma ocorrência entre os cinco grupos evidenciados. O Ministério da Ciência e Tecnologia e o $\mathrm{CNPq}$ também figuram entre os autores citados no grupo. 
O Gráfico 7 traz os autores mais citados nos artigos no GT6 (Informação, educação e trabalho) que contempla estudos sobre educação dos profissionais da informação e mercado de trabalho em informação.

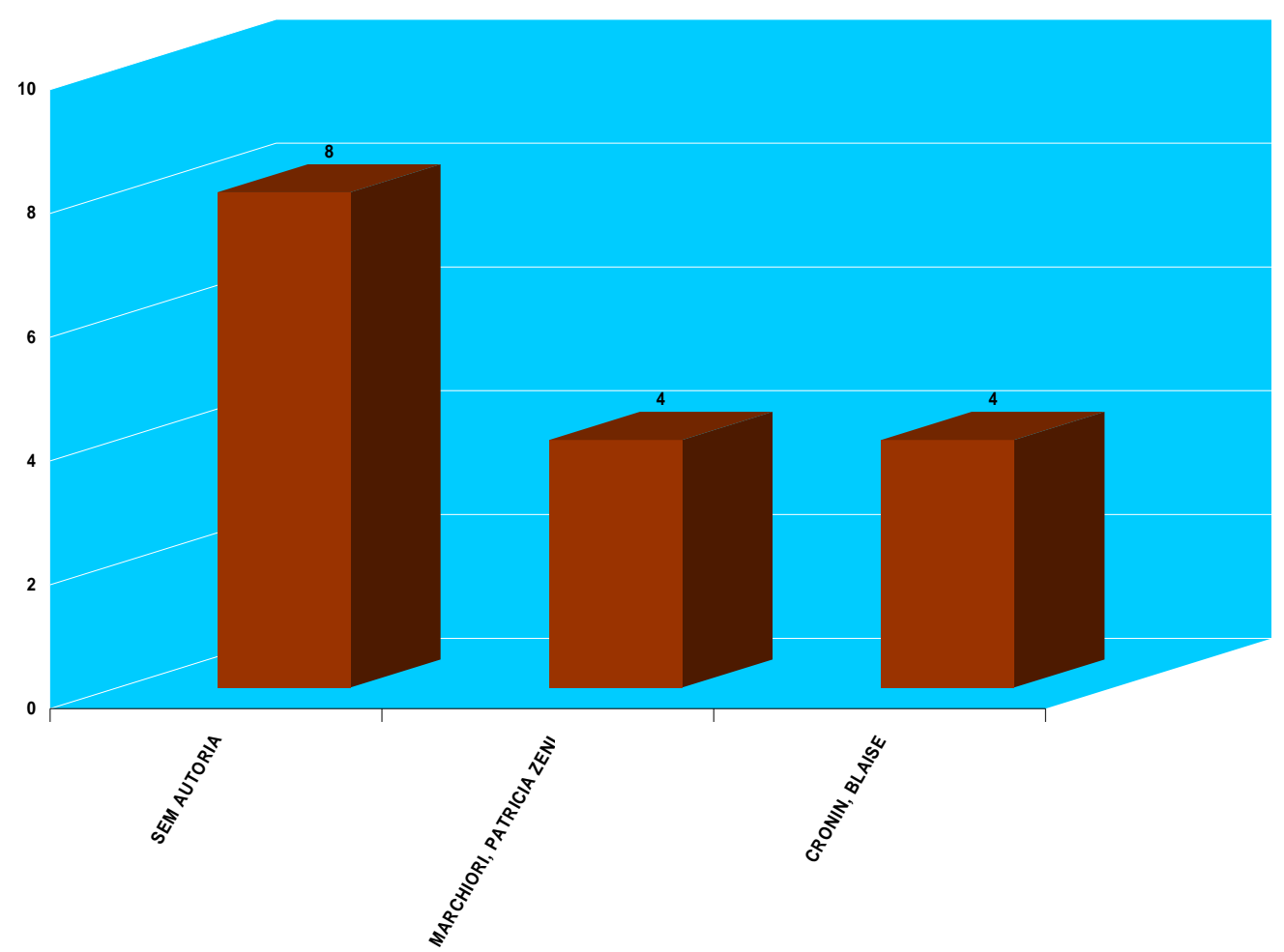

\section{GRÁFICO 7: AUTORES MAIS CITADOS NO GT6}

O que se observa na representação cartográfica acima é a presença do item Sem Autoria na primeira posição. Sua presença é visualizada nos seis grupos analisados, sempre ocupando a primeira colocação. A segunda é assumida pela pesquisadora brasileira Patrícia Zeni Marchiori, com 4 citações, como também por Blaise Cronin.

O gráfico apresenta algumas particularidades bem pontuais como a presença de somente duas pesquisadoras como as mais citadas. Como dito anteriormente, o estudo estabeleceu um recorte para as ocorrências de citações, sendo quatro o número mínimo. Assim, registra-se que o núcleo das citações sobre assuntos ligados à formação e prática profissional na Ciência da Informação é disperso e se restringe a poucos pesquisadores.

Tendo em vista as temáticas abarcadas pelo GT7 (Produção e comunicação da informação em CT\&I), o Gráfico 8 exibe os autores mais citados por este domínio da Ciência da Informação. 


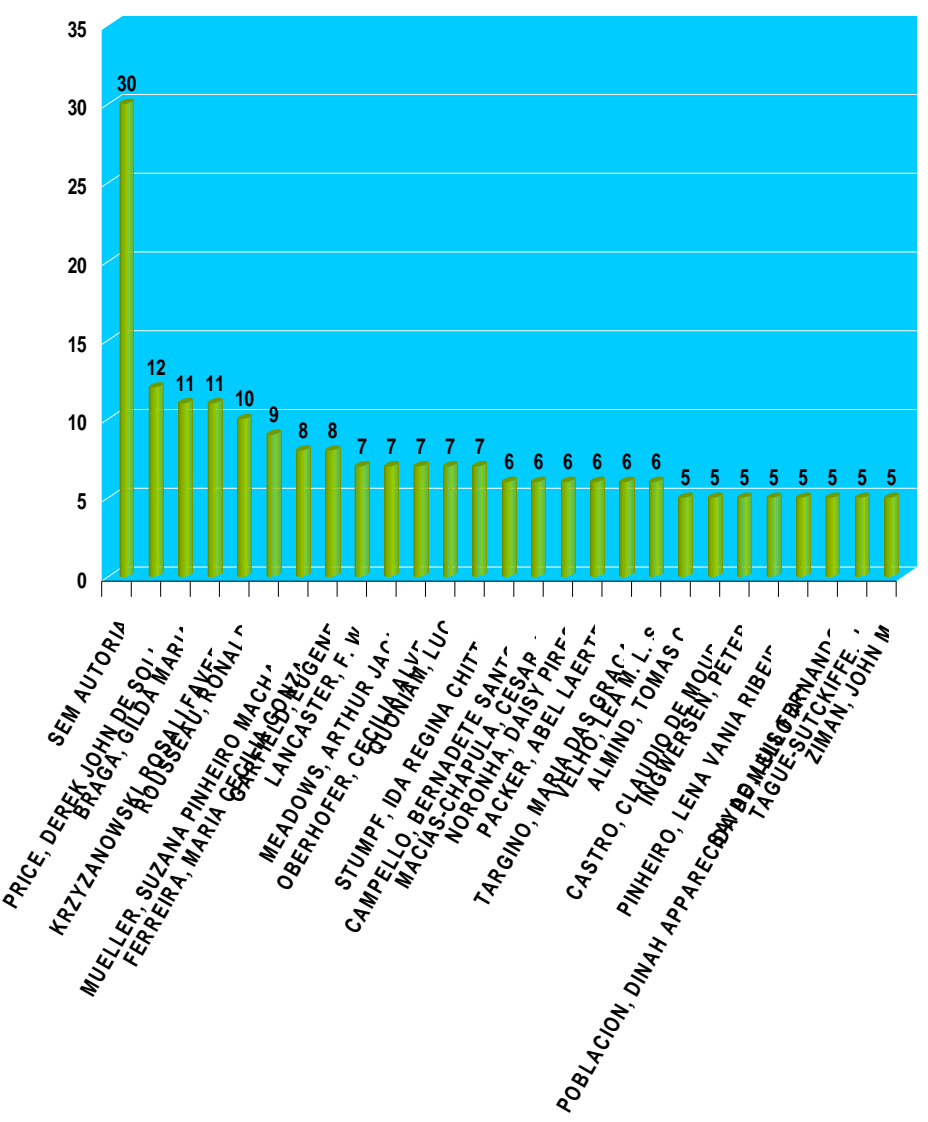

GRÁFICO 8: AUTORES MAIS CITADOS NO GT7

Um comentário inicial antes das análises é fundamental e tem a ver com o valor das ocorrências de citações, que neste grupo foi de número mínimo cinco. A diferença deste número neste grupo em relação aos demais se justifica porque o grupo temático em pauta apresentou um número de citações elevado, necessitando aumentar a seleção das citações em números absolutos para a apresentação dos pesquisadores mais citados na representação cartográfica. Contudo, cabe salientar que o aumento não traz distorções pelo número de citações contempladas pelo grupo que foi superior aos demais.

A avaliação inicial do Gráfico 8 é que o grupo de trabalho é o segundo que apresentou um número maior de pesquisadores brasileiros citados em relação aos estrangeiros. O Gráfico aponta o item Sem Autoria na primeira posição, com um número elevado de citações, num total de 30. A segunda posição é ocupada pelo físico americano Derek John de Solla Price, com 12 citações, seguido de duas brasileiras Gilda Maria Braga e Rosali Fávero Krzyzanowski, ambas com 11 citações. Em seguida, surge Ronald Rousseau, sendo citado 10 vezes, e depois, Suzana Pinheiro Machado Mueller, com 9 citações.

Do grupo de autores mais citados, nota-se a presença significativa de autores brasileiros. Entre os estrangeiros mais citados no grupo, destaque para Eugene Garfield, F. W. Lancaster, Arthur Jack Meadows, Luc Quoniam, César A. Macias-Chapula, Tomas C. Almind, Peter Ingwersen, J. Tague-Sutickffe e John Ziman. Destes autores destacados, F. W. Lancaster e Peter Ingwersen já figuraram em outros grupos de trabalhos como autores mais citados. 
A representação cartográfica aponta 14 brasileiros entre os mais citados, onde 11 destes são considerados atores científicos ligados à Ciência da Informação. Assim, evidencia-se Maria Cecília Gonzaga Ferreira, Cecília Alves Oberhofer, Ida Regina Chittó Stumpf, Bernadete Santos Campello, Daisy Pires Noronha, Maria das Graças Targino, Lena Vânia Ribeiro Pinheiro, Dinah Aparecida de Mello Aguiar Población e Luís Fernando Sayão. Os brasileiros ligados de outras áreas do conhecimento que receberam citações no âmbito deste grupo foram Abel Laerte Packer, Lea M. L. S. Velho e Cláudio de Moura Castro.

\section{A FRENTE DE PESQUISA DA CIÊNCIA DA INFORMAÇÃO NO BRASIL}

Após a exposição dos autores mais citados segundo os Grupos de Trabalho da ANCIB através de representações cartográficas, apresentar-se-á a frente de pesquisa da Ciência da Informação. O Quadro 2, exibido a seguir, traz os principais personagens científicos da Ciência da Informação identificados através das citações efetuadas nos 386 artigos veiculados pelo periódico Ciência da Informação, no período de 1995 a 2005.

\begin{tabular}{|c|c|c|}
\hline Grupo & \multicolumn{2}{|c|}{$\begin{array}{c}\text { Descrição do Grupo } \\
\end{array}$} \\
\hline GT1 & Estudos Históricos & ógicos da Informação \\
\hline Pesquisadores Brasileiros & \multicolumn{2}{|c|}{ Pesquisadores Estrangeiros } \\
\hline $\begin{array}{l}\text { Maria Nélida Gonzalez de Gomez } \\
\text { Aldo de Albuquerque Barreto } \\
\text { Solange Puntel Mustafa } \\
\text { Lena Vânia Pinheiro Ribeiro }\end{array}$ & $\begin{array}{c}\text { Tefko Saracevic } \\
\text { Gernot Wersig } \\
\text { Nicholas J. Belkin } \\
\text { Thomas S. Kuhn } \\
\text { Edgar Morin } \\
\text { H. Borko } \\
\text { Pierre Bourdieu } \\
\text { Karl Raymond Popper } \\
\text { Michel Foucault }\end{array}$ & $\begin{array}{c}\text { Boaventura de Sousa Santos } \\
\text { Gaston Bachelard } \\
\text { Ludwig Von Bertalanffy } \\
\text { B. C. Brookes } \\
\text { Jurgen Habermas } \\
\text { Peter Ingwersen } \\
\text { Bruno Latour } \\
\text { Pierre Levy } \\
\text { Robert S. Taylor }\end{array}$ \\
\hline GT 2 & \multicolumn{2}{|c|}{ Organização e Representação do Conhecimento } \\
\hline Pesquisadores Brasileiros & \multicolumn{2}{|c|}{ Pesquisadores Estrangeiros } \\
\hline $\begin{array}{c}\text { Maria Luiza de Almeida Campos } \\
\text { Murilo Bastos da Cunha } \\
\text { CNPq }\end{array}$ & $\begin{array}{l}\text { F. W. Lancaster } \\
\text { Ingetraut Dahlberg } \\
\text { Tefko Saracevic } \\
\text { Marcia J. Bates }\end{array}$ & $\begin{array}{l}\text { S. R. Ranganathan } \\
\text { Carol Tenopir } \\
\text { Derek Langridge } \\
\text { José Antonio Moreiro Gonzalez }\end{array}$ \\
\hline GT 3 & \multicolumn{2}{|c|}{ Mediação, Circulação e Uso da Informação } \\
\hline Pesquisadores Brasileiros & \multicolumn{2}{|c|}{ Pesquisadores Estrangeiros } \\
\hline $\begin{array}{c}\text { Aldo de Albuquerque Barreto } \\
\text { Regina Maria Marteleto } \\
\text { Nice Menezes de Figueiredo } \\
\text { Maria Nélida Gonzalez de Gomez } \\
\text { Isa Maria Freire } \\
\text { Sueli Mara Soares Pinto Ferreira } \\
\text { Murilo Bastos da Cunha } \\
\text { Bernadete Santos Campello } \\
\text { Elisabeth Adriana Dudziak }\end{array}$ & $\begin{array}{l}\text { F. W. Lancaster } \\
\text { Pierre Levy } \\
\text { Bem Shneiderman } \\
\text { Edward A. Fox } \\
\text { Brenda Dervin } \\
\text { Chun Wei Choo }\end{array}$ & $\begin{array}{c}\text { Vannevar Bush } \\
\text { Jakob Nielsen } \\
\text { Yves-François Le Coadic } \\
\text { Pierre Bourdieu } \\
\text { Ton D. Wilson }\end{array}$ \\
\hline GT 4 & \multicolumn{2}{|c|}{ Gestão da Informação e do Conhecimento nas Organizações } \\
\hline Pesquisadores Brasileiros & \multicolumn{2}{|c|}{ Pesquisadores Estrangeiros } \\
\hline $\begin{array}{c}\text { Kira Tarapanoff } \\
\text { Anna da Soledade Vieira } \\
\text { Murilo Bastos da Cunha } \\
\text { Aldo de Albuquerque Barreto } \\
\text { Kátia Maria Lemos Montalli } \\
\text { Antonio Lisboa C. de Miranda } \\
\text { Mônica Erichsen Nassif Borges } \\
\text { Gregório J. Varvakis } \\
\text { Waldomiro C. S. Vergueiro }\end{array}$ & $\begin{array}{l}\text { Michael E. Porter } \\
\text { Peter F. Drucker } \\
\text { Thomas H. Davenport } \\
\text { Hirotaka Takeuchi } \\
\text { Ikujiro Nonaka } \\
\text { Laurence Prusak } \\
\text { Chun Wei Choo } \\
\text { Leonard M. Fuld }\end{array}$ & $\begin{array}{c}\text { Manuel Castells } \\
\text { P. Kotler } \\
\text { Blaise Cronin } \\
\text { Thomas A. Stewart } \\
\text { Arão Shapiro } \\
\text { David A. Garvin } \\
\text { Idalberto Chiavenato } \\
\text { F. W. Lancaster }\end{array}$ \\
\hline
\end{tabular}

Enc. Bibli: R. Eletr. Bibliotecon. Ci. Inf., Florianópolis, n. 26, $2^{\circ}$ sem.2008 


\begin{tabular}{|c|c|c|}
\hline \multicolumn{3}{|c|}{ Política e Economia da Informação } \\
\hline \multicolumn{2}{|c|}{ Pesquisadores Brasileiros } & Pesquisadores Estrangeiros \\
\hline $\begin{array}{l}\text { Antonio Lisboa C. de Miranda } \\
\text { José Maria Jardim } \\
\text { CNPq } \\
\text { Ministério de Ciência e Tecnologia } \\
\text { Aldo de Albuquerque Barreto }\end{array}$ & $\begin{array}{c}\text { Kira Tarapanoff } \\
\text { Helena Maria Martins Lastres } \\
\text { Maria Nélida Gonzalez de Gomez } \\
\text { Vânia Maria R. Hermes de Araújo } \\
\text { Anna da Soledade Vieira } \\
\text { Marta Pinheiro Aun }\end{array}$ & $\begin{array}{l}\text { Manuel Castells } \\
\text { Ioneji Masuda } \\
\text { Pierre Levy } \\
\text { Jurgen Habermas } \\
\text { C. Freeman } \\
\text { Hugo Assmann }\end{array}$ \\
\hline GT 6 & \multicolumn{2}{|c|}{ Informação, Educação e Trabalho } \\
\hline Pesquisadores Brasi & \multicolumn{2}{|c|}{ Pesquisadores Estrangeiros } \\
\hline Patrícia Zeni Marc & & laise Cronin \\
\hline GT 7 & \multicolumn{2}{|c|}{ Produção e Comunicação da Informação em CT\&I } \\
\hline \multicolumn{2}{|c|}{ Pesquisadores Brasileiros } & Pesquisadores Estrangeiros \\
\hline $\begin{array}{c}\text { Gilda Maria Braga } \\
\text { Rosali Fávero Krzyzanowski } \\
\text { Suzana Pinheiro Machado Mueller } \\
\text { Maria Cecília Gonzaga Ferreira } \\
\text { Cecília Alves Oberhofer } \\
\text { Ida Regina Chittó Stumpf } \\
\text { Bernadete Santos Campello } \\
\text { Daisy Pires Noronha }\end{array}$ & $\begin{array}{c}\text { Abel Laerte Packer } \\
\text { Maria das Graças Targino } \\
\text { Léa M. L. S. Velho } \\
\text { Cláudio de Moura Castro } \\
\text { Lena Vânia Ribeiro Pinheiro } \\
\text { Dinah Ap. de M. A. Población } \\
\text { Luís Ferando Sayão }\end{array}$ & $\begin{array}{c}\text { Derek John de Solla Price } \\
\text { Ronald Rosseau } \\
\text { Eugene Garfield } \\
\text { F. W. Lancaster } \\
\text { Arthur Jack Meadows } \\
\text { Luc Quoniam } \\
\text { César A. Macias-Chapula } \\
\text { Tomas C. Almind } \\
\text { Peter Ingwersen } \\
\text { J. Tague-Sutckiffe } \\
\text { John M. Ziman }\end{array}$ \\
\hline
\end{tabular}

\section{COMENTÁRIOS FINAIS}

As principais considerações sobre os resultados obtidos ao longo do período analisado são apresentadas, a seguir:

- As médias de citações por artigos de periódicos revelam um fato peculiar ao determinado por Price (1965). A média de citações por artigos revela pela presente pesquisa foi de 19,44, superior ao que o autor menciona em seu estudo. Já a média de artigos citados é inferior ao levantado por ele, com 7,08 artigos citados, evidenciando assim que o livro também é importante para área, confirmando a natureza da Ciência da Informação como uma ciência social aplicada;

- A Ciência da informação carece de instrumentos de classificação que considerem os diversos espaços e possibilidades de atuação. Mesmo utilizando uma divisão legitimada pela comunidade como os Grupos de Trabalho da ANCIB, a referida divisão apresenta problemas quanto aos seus limites territoriais;

- Os Grupos de Trabalho 3 e 4 da ANCIB, conhecidos como Mediação, Circulação e Uso da Informação e Gestão da Informação e do Conhecimento nas Organizações, respectivamente, possuem ementas abrangentes, provocando distorções nas interpretações e categorizações dos trabalhos desenvolvidos no âmbito da área;

- Sobre a ocorrência de citações dos autores por grupo de pesquisa, as representações cartográficas sinalizam a frente de pesquisa formada a partir do periódico analisado. Contudo, é importante registrar que tal pesquisa analisou um periódico, cabendo assim novas pesquisas aos outros periódicos nacionais para a obtenção de um panorama mais completo da frente de pesquisa através dos periódicos nacionais;

- Das representações cartográficas apresentadas, percebe-se que alguns autores conseguem transitar em mais de um grupo de trabalho, o que pode demonstrar a condição destes autores pertencerem ao grupo de elite, como afirma Price (1965). 
Espera-se assim, que esta contribuição tenha contribuído para o entendimento dos caminhos percorridos pela Ciência da Informação, como também para o fortalecimento das questões metodológicas ligadas aos estudos métricos da informação. Enfatiza-se que o estudo serve de parâmetro para o delineamento e desenvolvimento de novas pesquisas que se relacionam com a formulação de indicadores científicos, no mesmo instante que se coloca como mais um trabalho de sinalização do estágio alcançado pela Ciência da Informação no Brasil.

\section{REFERÊNCIAS}

BUFREM, L.; PRATES, Y. O saber científico e as práticas de mensuração da informação. Ciência da Informação, Brasília, v. 34, n. 2, p. 9-25, 2005.

CUNHA, M. V. da. Os periódicos em ciência da informação: uma análise bibliométrica. Ciência da Informação, Brasília, v. 14, n. 1, p. 37-45, 1985.

FORESTI, N. A. B. Contribuição das revistas brasileiras de Biblioteconomia e Ciência da Informação enquanto fonte de referência para a pesquisa. Ciência da Informação, Brasília, v. 19, n. 1, p. 53-71, 1990.

GOMES, M. Y. F. S. de F. Tendências atuais da produção científica em Biblioteconomia e Ciência da Informação no Brasil. Datagramazero, v. 7, n. 3, 2006. Disponível em:<www.dgz.org.br>. Acesso em: 25 dez. 2006.

MUELLER, S. P. M. O círculo vicioso que prende os periódicos nacionais. Datagramazero, n. 0, dez. 1999. Disponível em: <www.dgz.org.br>. Acesso em: 23 nov. 2004.

NORONHA, D. P.; FERREIRA, S. M. S. P. Índices de citação. In: CAMPELLO, B. S.; CENDÓN, B. V.; KREMER, J. M. (Org.). Fontes de informação para pesquisadores e profissionais. Belo Horizonte: Ed. da UFMG, 2003. p. 246-262.

OLIVEIRA, M. de; SANTANA, M. A. L. V ENANCIB: análise dos caminhos de pesquisa. In: ENCONTRO NACIONAL DE PESQUISA EM CIÊNCIA DA INFORMAÇÃO, 6., 2005, Florianópolis. Anais eletrônicos... Florianópolis: ANCIB, 2005.

PARLEMITI, R; POLITY, Y. Dynamiques de l'institucionnlisation sociale et cognitive dês sciences de l'information. In: BOURE, R. (Ed.). Les origines dês Sciences de l'information et de la communication: regards croisés. Paris: PUS, 2002, p. 95-123.

PINHEIRO, L. V. R. Processo evolutivo e tendências contemporâneas da Ciência da Informação. Informação \& Sociedade: Estudos, João Pessoa, v. 15, n. 1, p. 1-21, 2005.

PRICE, D. J. de S. Networks of scientific papers: the pattern of bibliographic references indicates the nature of the scientific research front. Science, [Washington, D.C.], v. 149, n. 3.683, p. 510-515, 1965.

QUEIROZ, F. M.; NORONHA, D. P. Temáticas das dissertações e teses em Ciência da Informação no programa de pós-graduação em Ciências da Comunicação da USP. Ciência da Informação, Brasília, v. 33, n. 2, p. 132-142, 2004. 
SILVEIRA, M. A. A. da; MOURA, A. M. S. de. O artigo científico como fonte de informação utilizada nos anais do SNBU. In: SEMINÁRIO NACIONAL DE BIBLIOTECAS UNIVERSITÁRIAS, 14., 2006, Salvador. Anais eletrônicos... Salvador: CBBU, 2006.

TARGINO, M. das G.; GARCIA, J. C. R. Ciência brasileira na base de dados do Institute for Scientific Information (ISI). Ciência da Informação, Brasília, v. 29, n. 1, p. 103-107, 2000.

VANTI, N. A. P. Da bibliometria à webometria: uma exploração conceitual dos mecanismos utilizados para medir o registro da informação e a difusão do conhecimento. Ciência da Informação, Brasília, v. 31, n. 2, p. 152-162, 2002.

WHITLEY, R. Cognitive and social institucionalization of scientific specialities and research areas. In: WHITLEY, R. (Ed.). Social processes of scientific development. London: Routledge and Kegan, 1974. p. 69-95.

WHITLEY, R. The context of scientific investigation. In: KNORR, Karin D.; KROHN, Roger; WHITLEY, Richard. The social process of scientific investigation. London: D. Reidel, 1980. p. 297-321. v. 4.

\begin{abstract}
Identification of the main scientific characters connected with the brazilian Information Science, through the Ciência da Informação journal. The main objective is to investigate the social institutionalization of information science with a basis on citations published in the Ciencia da Informação journal between 1995 and 2005. It users the scienciometric method, through the citation analysis and cartographic representation. The results are presented according to work groups of the ANCIB. It considers the results and suggests some modifications to the citations indications.
\end{abstract}

KEYWORDS: Information Science. Research Front. Scienciometric. Ciência da Informação Journal. Citation Studies. Institutionalization of the Scientific Research.

Originais recebidos em: 15/02/2008

Texto aprovado em: 02/09/2008 